\title{
Inclusion Measure and Similarity Measure of Intuitionistic and Interval-valued Fuzzy Sets
}

\author{
Hongying Zhang ${ }^{1}$ Minggao Dong ${ }^{2}$ Wenxiu Zhang ${ }^{1}$ Xiaoxue Song ${ }^{3}$ \\ ${ }^{1}$ Institute for Information and System Sciences, Faculty of Science, Xi'an Jiaotong University, \\ Xi'an 710049, P. R. China \\ ${ }^{2}$ School of Economy and Management, Xi'an Shiyou University \\ ${ }^{3}$ Xianyang Normal College
}

\begin{abstract}
We begin the paper studying the axioms that the inclusion measure of an element in another element of a lattice and the similarity measure between two elements should satisfy. Next, we present a series of expressions of inclusion measures, first for intuitionistic values and then for intuitionistic fuzzy sets ( or interval-valued, since they have a strong connection), discussing in both cases their properties. Then we discuss similarity measure which can be transformed by the proposed inclusion measure based on their axiomatic definitions. The similarity measures defined by the proposed inclusion measures of IFSs are seem reasonable.
\end{abstract}

Keywords: Inclusion measure, Similarity measure, Intuitionistic value, Intuitionistic fuzzy set, Interval-valued fuzzy set

\section{Introduction}

Since fuzzy set was introduced by Zadeh [1], many new approaches and theories treating imprecision and uncertainty have been proposed. Intuitionistic fuzzy sets (IFSs)[2] and interval-valued fuzzy sets (IVFSs) [3]-[5] are two important intuitively straightforward extensions, which were introduced independently to alleviate some of the drawbacks of fuzzy set theory. IFSs assign to each element of the universe both a degree of membership $\mu$ and one of non-membership $\nu$ such that $\mu+\nu \leq 1$, thus relaxing the enforced duality $\nu=1-\mu$ from fuzzy set theory. IVFS theory emerged from the observation that in a lot of cases, no objective procedure is available to select the crisp membership degrees of elements in a fuzzy set. It was suggested to alleviate that problem by allowing to specify only an interval $\left[\mu_{1}, \mu_{2}\right]$ to which the actual membership degree is assumed to belong. IFS theory and IVFS theory make descriptions of the objective world more realistic, practical, and accurate, making it very promising. They have been widely applied in different fields of science, for example De et al. [6] in medical diagnosis, Gorzalczany [7] and Bustince [8] in approximate reasoning, Turksen [9] and Cornelis et al. [10] in interval-valued and intuitionistic logic, etc..

Inclusion measure and similarity measure of fuzzy sets are two important topics in fuzzy set theory. An inclusion measure indicates the degree to which a given fuzzy set is contained in another fuzzy set. Many researchers have tried to capture this intuition by constructive approach [11]-[14] and axiomatic approach [15]-[17]. In paper [15], Sinha and Dougherty first listed nine properties that a reasonable inclusion measure should have and then proceeded to derive inclusion measures which have these properties. Young and Cornelis et al. gave the general form of axioms for an inclusion measure in [16] and [17]. On the other hand, the similarity measure is employed to determine whether a model or a rule should be matched with the expectation in pattern recognition and rule matching. Many authors have paid much attention to the theoretic study and application of fuzzy similarity measure. Pappis et al. have issued a series of papers [18]-[20] which take an axiomatic view to similarity measures. Liu [21] proposed an alternative set of axioms which is applied widely. In [22], Fan gives axioms for entropy, distance and $\sigma$ similarity. Wang et al. criticized Pappis' work in [23], present a modified definition of similarity.

Some authors have investigated inclusion measure and similarity measure of IFSs and IVFSs. $H$. Bustince [24] introduced the interval-valued indicator of inclusion grade for interval-valued fuzzy sets which is appropriate for approximate reasoning. But that indicator is not coincide with the one proposed by Sinha et al. in fuzzy mathematical morphology. C. Cornelis and E. E. Kerre [25] gave the intuitionistic-valued inclusion measures of IFSs. 
Grzgorzewski and E. Mrowka [26] gave the systems of axioms, characterizing the inclusion measure (subsethood measure) of intuitionistic fuzzy sets, which is close to that given by Young than by Sinha and Dougherty. However, the list of the axioms is too strict with the inclusion measure of an IFS, the inclusion measure based on the Hamming distance between IFSs suggested in this paper did not satisfy all of the axioms. J. H. Park, J. S. Park and Y. C. Kwun [27] defined and studied an kind of sophisticated inclusion measure of IFSs and applied it to interval-valued fuzzy topological spaces. Zhang and $\mathrm{Fu}[28]$ discussed the similarity measures on L-fuzzy sets. Li, Olson and Zheng [29] gave the comparative analysis of similarity measures between IFSs and the positive aspects of each similarity measure were demonstrated. In this paper, we want to introduce an axiom definition of inclusion measure of IFSs and IVFSs, put forward some formulas to calculate inclusion measure and discuss the relation of the inclusion measure and similarity measure.

The rest of our work is organized as follows. In section 2, we recall some basic notions of $I F S s$ and $I V F S s$. In section 3, we introduce the concept of HM inclusion measure of two elements of a lattice and discuss the inclusion measure of two intuitionistic values and two IFSs; In section 4, the axiomatic definition of $\mathbb{S}$ similarity measure is proposed and a theorem is given to show that similarity measure can be transformed by the HM inclusion measure; The final section is conclusion.

\section{IFS and IVFS theory}

Throughout this paper, $X=\left\{x_{1}, \ldots, x_{n}\right\}$ is the universal set; $\mathscr{P}(X)$ and $\mathscr{F}(X)$ are the sets of all the crisp set and fuzzy sets in $X$ respectively; $\mathrm{D}([0,1])$ is the set of all the closed subintervals of the interval $[0,1] . \quad\left[\frac{1}{2}\right]_{X}$ is the fuzzy set of $\mathrm{X}$ for which $\left[\frac{1}{2}\right]_{X}(x)=\frac{1}{2}, \forall x \in X ; A_{c}$ is the complement of fuzzy set A where $A_{c}(x)=1-A(x)$.

\subsection{Definition, operations on $\operatorname{IFSs}(X)$}

Definition 1. [10] Intuitionistic value lattice $\left(L^{*}, \leq_{L^{*}}\right)$ is a lattice defined on the intuitionistic value set $L^{*}=\left\{(\mu, \nu) \in[0,1]^{2} \mid \mu+\nu \leq 1\right\}$ endowed with the following partial order:

$$
\left(\mu_{1}, \nu_{1}\right) \leq_{L^{*}}\left(\mu_{2}, \nu_{2}\right) \Leftrightarrow \mu_{1} \leq \mu_{2}, \nu_{1} \geq \nu_{2}
$$

The operators $\wedge$ and $\vee$ on $\left(L^{*}, \leq_{L^{*}}\right)$ are defined as follows, for $\left(\mu_{1}, \nu_{1}\right),\left(\mu_{2}, \nu_{2}\right) \in L^{*}$ :

$$
\begin{aligned}
& \left(\mu_{1}, \nu_{1}\right) \wedge\left(\mu_{2}, \nu_{2}\right)=\left(\min \left\{\mu_{1}, \mu_{2}\right\}, \max \left\{\nu_{1}, \nu_{2}\right\}\right) \\
& \left(\mu_{1}, \nu_{1}\right) \vee\left(\mu_{2}, \nu_{2}\right)=\left(\max \left\{\mu_{1}, \mu_{2}\right\}, \min \left\{\nu_{1}, \nu_{2}\right\}\right) .
\end{aligned}
$$

The lattice $\left(L^{*}, \leq_{L^{*}}\right)$ is a complete lattice: for each $A \subseteq L^{*}$,

$$
\begin{aligned}
& \sup A=(\sup \{\mu\}, \inf \{\nu\}), \forall(\mu, \nu) \in A, \\
& \inf A=(\inf \{\mu\}, \sup \{\nu\}), \forall(\mu, \nu) \in A .
\end{aligned}
$$

Definition 2. Let $(L, \leq)$ be a complete lattice and $\varphi: L \rightarrow L$ a mapping from $L$ to itself. The mapping $\varphi$ is called an order-reversing involution if following conditions are satisfied:

(1) $\varphi(\varphi(a))=a, \forall a \in L$

(2) $a \leq b \Rightarrow \varphi(b) \leq \varphi(a), \forall a, b \in L$.

In the following paper, we define $c(a)=a^{c}=$ $1-a, \forall a \in L$, then $\mathrm{c}$ is a order-reversing involution on L. For any $(\mu, \nu) \in\left(L^{*}, \leq_{L^{*}}\right),(\mu, \nu)^{c}=(\nu, \mu)$.

Definition 3. [2] An intuitionistic fuzzy set in $\mathrm{X}$ is an expression A given by

$$
A=\left\{<x, \mu_{A}(x), \nu_{A}(x)>\mid x \in X\right\}
$$

where

$$
\mu_{A}: X \rightarrow[0,1], \quad \nu_{A}: X \rightarrow[0,1]
$$

with the condition $0 \leq \mu_{A}(x)+\nu_{A}(x) \leq 1$ for all $x \in X$. The set of all the IFSs in $\mathrm{X}$ is denoted by $\operatorname{IFS} s(X) ;(a, b)_{X}$ is the IFS of $\mathrm{X}$ for which $(a, b)_{X}(x)=(a, b), \forall x \in X$.

The following expressions are defined in [2] for all $A, B \in \operatorname{IFSs}(X)$ :

(1) $A \leq B$ if only if $\mu_{A}(x) \leq \mu_{B}(x)$ and $\nu_{A}(x) \geq \nu_{B}(x)$ for all $x \in X$,

(2) $A \preccurlyeq B$ if only if $\mu_{A}(x) \leq \mu_{B}(x)$ and $\nu_{A}(x) \leq \nu_{B}(x)$ for all $x \in X$,

(3) $A=B$ if only if $A \leq B$ and $B \leq A$,

(4) $A^{c}=\left\{<x, \nu_{A}(x), \mu_{A}(x)>\mid x \in X\right\}$.

It's obvious that $(\operatorname{IFSs}(X), \leq)$ is a lattice.

\subsection{Definition, operations on $\operatorname{IVFSs}(X)$}

Definition 4. [5] An interval-valued fuzzy set in $\mathrm{X}$ is an expression $\mathrm{A}$ given by

$$
A=\{<x, A(x)>\mid x \in X\}
$$

where

$$
A: X \rightarrow D([0,1])
$$




$$
x \rightarrow A(x)=\left[A_{-}(x), A_{+}(x)\right] \in D([0,1]) .
$$

For simplicity, we denote $A=\left[A_{-}, A_{+}\right]$. The set of all the $I V F S s$ in $\mathrm{X}$ is denoted with $I V F S s(X)$.

The following expressions are defined in [5], [30] for all $A, B \in I V F S s(X)$ :

(1) $A \leq B$ if only if $A_{-}(x) \leq B_{-}(x)$ and $A_{+}(x) \leq B_{+}(x)$ for all $x \in X$, $x \in X$

(2) $A \lesssim B$ if only if $B_{-}(x) \leq A_{+}(x)$ for all

(2) $A=B$ if only if $A \leq B$ and $B \leq A$,

(3) $A^{c}=\left[A_{+}^{c}, A_{-}^{c}\right]$.

\subsection{Relation between $\operatorname{IFSs}(X)$ and $\operatorname{IVFSs}(X)$}

In 1989, Atanassov and Gargov [31] using

(a) the map $f$ assigns to every $I V F S s(X) A$ and $\operatorname{IFSs}(X) B, B=f(A)$ given by

$\mu_{A}(x)=A_{-}(x), \quad \nu_{B}(x)=1-A_{+}(x) ;$

(b) the map $g$ assigns to every $I F S s(X) B$ and $\operatorname{IVFSs}(X) A, A=g(B)$ given by

$$
A(x)=\left[\mu_{B}(x), 1-\nu_{B}(x)\right]
$$

proved that $I F S s(X) \quad B$ and $I V F S s(X) \quad A$ are equipollent generalizations of the notion of $F S s(X)$. Although $I F S s(X)$ and $I V F S s(X)$ have arisen on different ground and they have different semantics, sometimes they are even mathematically equivalent. So these approaches are in general not independent and there exist a strong connection between intuitionistic fuzzy sets and interval-valued fuzzy sets.

Taking this fact into account all the definitions, propositions and theorems we will do from now on for the $\operatorname{IFS} S(X)$, can be extended to the $\operatorname{IVFSs}(X)$.

\section{Inclusion measure of IFSs}

C. Cornelis and E.E. Kerre [25] first discussed the inclusion measure for intuitionistic fuzzy sets. They defined the inclusion measure by an intuitionistic value not by a real number in $[0,1]$ and suggested a set of axioms for such measure, based on the SinhaDougherty axioms. The application showed that this intuitionistic-valued inclusion measure was appropriate for approximate reasoning. But the set of C. Cornelis and E.E. Kerre's degree of measuring subsethood is still a partial order set. Grzgorzewski and E. Mrowka [26] gave the systems of axioms, characterizing the numerical inclusion measure (subsethood measure) of intuitionistic fuzzy sets, which is close to that given by Young [16]. However the example given in [26] did not satisfy all of the axioms proposed by Grzgorzewski and E. Mrowka. So just like what Young [16] and Fan [32] have done, we define a kind of hybrid monotonic inclusion measure, which indicates the degree to which an element is contained in another element of a complete lattice, by describing the main characteristics of an inclusion measure postulated by Sinha and Dougherty [15].

Definition 5. Let $(L, \leq)$ be a complete lattice, we denote the mapping Inc: $L \times L \rightarrow[0,1]$ as an hybrid monotonic inclusion measure on lattice $\mathrm{L}$, such that the value Inc $(a, b)$ will indicate to what extent an element of $\mathrm{L}$ included in another element of $\mathrm{L}$ and satisfies the following conditions:

(HM1) $0 \leq \operatorname{Inc}(a, b) \leq 1, \forall a, b \in L$;

(HM2) $\operatorname{Inc}(a, b)=0$ if $a=[1], b=[0]$ where $[0]$ and [1] are the minimum element and maximum element of $\mathrm{L}$ respectively;

(HM3) Inc $(a, b)=1 \Leftrightarrow a \leq b, \forall a, b \in L$;

(HM4) If $a \leq b$, for all $c \in L$, then $\operatorname{Inc}(c, a) \leq$ $\operatorname{Inc}(c, b)$ and $\operatorname{Inc}(b, c) \leq \operatorname{Inc}(a, c)$.

For simplicity, we call the hybrid monotonic inclusion measure as HM inclusion measure. For $\quad\left(L^{*}, \leq_{L^{*}}\right), \quad[0]=(0,1),[1]=(1,0), \quad$ while for $(\operatorname{IFSs}(X), \leq),[0]=\{(x,(0,1)), \mid \forall x \in X\},[1]=$ $\{(x,(1,0)), \mid \forall x \in X\}$.

\subsection{Inclusion measure of intu- itionistic value}

Lemma 1. If $a, b, c$ and $d$ are positive real number, then we have:

$$
\frac{a-b}{a+b} \geq \frac{c-d}{c+d} \Leftrightarrow a d \geq b c .
$$

Theorem 1. Let $\left(\mu_{1}, \nu_{1}\right),\left(\mu_{2}, \nu_{2}\right) \in\left(L^{*}, \leq_{L^{*}}\right)$ be two intuitionistic values, then

$$
\begin{gathered}
\operatorname{Inc}_{1}\left(\left(\mu_{1}, \nu_{1}\right),\left(\mu_{2}, \nu_{2}\right)\right)= \\
\left\{\begin{array}{cr}
0.5\left[\frac{\mu_{2}-\mu_{1}+\nu_{1}-\nu_{2}}{\left|\mu_{2}-\mu_{1}\right|+\left|\nu_{1}-\nu_{2}\right|}+1\right] & \text { else } \\
1 & \mu_{1}=\mu_{2}, \nu_{1}=\nu_{2} .
\end{array}\right.
\end{gathered}
$$

is a HM inclusion measure on Intuitionistic value lattice $\left(L^{*}, \leq_{L^{*}}\right)$.

Proof . It's obvious that $\operatorname{Inc}_{1}\left(\left(\mu_{1}, \nu_{1}\right),\left(\mu_{2}, \nu_{2}\right)\right)$ satisfies the conditions HM1, HM2 and Hm3, now we prove it have the third condition. 
(HM4) If $\left(\mu_{1}, \nu_{1}\right) \leq\left(\mu_{2}, \nu_{2}\right)$, for all $\left(\mu_{3}, \nu_{3}\right) \in$ $\left(L^{*}, \leq_{L^{*}}\right)$,

$$
\begin{gathered}
\operatorname{Inc}_{1}\left(\left(\mu_{1}, \nu_{1}\right),\left(\mu_{3}, \nu_{3}\right)\right)= \\
\left\{\begin{array}{cr}
0.5\left[\frac{\mu_{3}-\mu_{1}+\nu_{1}-\nu_{3}}{\left|\mu_{3}-\mu_{1}\right|+\left|\nu_{1}-\nu_{3}\right|}+1\right] & \text { else } \\
1 & \mu_{1}=\mu_{3}, \nu_{1}=\nu_{3} .
\end{array}\right.
\end{gathered}
$$

$\operatorname{Inc}_{1}\left(\left(\mu_{2}, \nu_{2}\right),\left(\mu_{3}, \nu_{3}\right)\right)=$

$$
\left\{\begin{array}{lr}
0.5\left[\frac{\mu_{3}-\mu_{2}+\nu_{2}-\nu_{3}}{\left|\mu_{3}-\mu_{2}\right|+\left|\nu_{2}-\nu_{3}\right|}+1\right] & \text { else } \\
1 & \mu_{2}=\mu_{3}, \nu_{2}=\nu_{3} .
\end{array}\right.
$$

We discuss them as follows:

(a) If $\mu_{1} \leq \mu_{3}, \nu_{1} \geq \nu_{3}$, then we have

$\operatorname{Inc}_{1}\left(\left(\mu_{1}, \nu_{1}\right),\left(\mu_{3}, \nu_{3}\right)\right)=1 \geq \operatorname{Inc}_{1}\left(\left(\mu_{2}, \nu_{2}\right),\left(\mu_{3}, \nu_{3}\right)\right)$;

(b) If $\mu_{3} \leq \mu_{2}, \nu_{2} \geq \nu_{3}$, then we have

$\operatorname{Inc}_{1}\left(\left(\mu_{2}, \nu_{2}\right),\left(\mu_{3}, \nu_{3}\right)\right)=0 \leq \operatorname{Inc}_{1}\left(\left(\mu_{1}, \nu_{1}\right),\left(\mu_{3}, \nu_{3}\right)\right)$;

(c) If $\mu_{2} \geq \mu_{1} \geq \mu_{3}, \nu_{1} \geq \nu_{2} \geq \nu_{3}$, then we get

$\operatorname{Inc}_{1}\left(\left(\mu_{1}, \nu_{1}\right),\left(\mu_{3}, \nu_{3}\right)\right)=0.5\left[\frac{\nu_{1}-\nu_{3}-\left(\mu_{1}-\mu_{3}\right)}{\nu_{1}-\nu_{3}+\left(\mu_{1}-\mu_{3}\right)}+1\right]$,

$\operatorname{Inc}_{1}\left(\left(\mu_{2}, \nu_{2}\right),\left(\mu_{3}, \nu_{3}\right)\right)=0.5\left[\frac{\nu_{2}-\nu_{3}-\left(\mu_{2}-\mu_{3}\right)}{\nu_{2}-\nu_{3}+\left(\mu_{2}-\mu_{3}\right)}+1\right]$, and $\left(\nu_{1}-\nu_{3}\right)\left(\mu_{2}-\mu_{3}\right) \geq\left(\nu_{2}-\nu_{3}\right)\left(\mu_{1}-\mu_{3}\right)$. Then by Lemma 1, we have

$$
\frac{\nu_{1}-\nu_{3}-\left(\mu_{1}-\mu_{3}\right)}{\nu_{1}-\nu_{3}+\left(\mu_{1}-\mu_{3}\right)} \geq \frac{\nu_{2}-\nu_{3}-\left(\mu_{2}-\mu_{3}\right)}{\nu_{2}-\nu_{3}+\left(\mu_{2}-\mu_{3}\right)},
$$

namely,

$$
\operatorname{Inc}_{1}\left(\left(\mu_{1}, \nu_{1}\right),\left(\mu_{3}, \nu_{3}\right)\right) \geq \operatorname{Inc}_{1}\left(\left(\mu_{2}, \nu_{2}\right),\left(\mu_{3}, \nu_{3}\right)\right) ;
$$

(d) When $\mu_{1} \leq \mu_{2} \leq \mu_{3}, \nu_{2} \leq \nu_{1} \leq \nu_{3}$, we can get the conclusion by the same way of (c).

Therefore $\operatorname{Inc}_{1}\left(\left(\mu_{1}, \nu_{1}\right),\left(\mu_{2}, \nu_{2}\right)\right)$ is a HM inclusion measure.

We can get the following corollary by Theorem 1.

Corollary 1. Let $\left(\mu_{1}, \nu_{1}\right),\left(\mu_{2}, \nu_{2}\right)$ be two intuitionistic values, then $\operatorname{Inc}_{1}\left(\left(\mu_{1}, \nu_{1}\right),\left(\mu_{2}, \nu_{2}\right)\right)$ has the following properties:

(I1) $\operatorname{Inc}_{1}\left(\left(\left(\mu_{1}, \nu_{1}\right)^{c},\left(\mu_{2}, \nu_{2}\right)\right)^{c}\right)=$

$$
\operatorname{Inc}_{1}\left(\left(\mu_{2}, \nu_{2}\right),\left(\mu_{1}, \nu_{1}\right)\right) ;
$$

(I2) $\operatorname{Inc}_{1}\left(\left(\mu_{1}, \nu_{1}\right),\left(\mu_{2}, \nu_{2}\right)\right)+\operatorname{Inc} c_{1}\left(\left(\mu_{2}, \nu_{2}\right),\left(\mu_{1}, \nu_{1}\right)\right)$ $=1$;

(I3) $\operatorname{Inc}_{1}\left(\left(\mu_{1}, \nu_{1}\right) \vee\left(\mu_{2}, \nu_{2}\right),\left(\mu_{3}, \nu_{3}\right)\right) \leq$

$$
\operatorname{Inc}_{1}\left(\left(\mu_{1}, \nu_{1}\right),\left(\mu_{3}, \nu_{3}\right)\right) \wedge \operatorname{Inc} c_{1}\left(\left(\mu_{2}, \nu_{2}\right),\left(\mu_{3}, \nu_{3}\right)\right) ;
$$

(I4) $\operatorname{Inc}_{1}\left(\left(\mu_{1}, \nu_{1}\right),\left(\mu_{2}, \nu_{2}\right) \wedge\left(\mu_{3}, \nu_{3}\right)\right) \leq$

$$
\operatorname{Inc}_{1}\left(\left(\mu_{1}, \nu_{1}\right),\left(\mu_{2}, \nu_{2}\right)\right) \wedge \operatorname{Inc} c_{1}\left(\left(\mu_{1}, \nu_{1}\right),\left(\mu_{3}, \nu_{3}\right)\right) .
$$

Remark 1. The equalities

$\operatorname{Inc}_{1}\left(\left(\mu_{1}, \nu_{1}\right) \wedge\left(\mu_{2}, \nu_{2}\right),\left(\mu_{3}, \nu_{3}\right)\right)=$

$$
\operatorname{Inc}_{1}\left(\left(\mu_{1}, \nu_{1}\right),\left(\mu_{2}, \nu_{2}\right)\right) \wedge \operatorname{Inc} c_{1}\left(\left(\mu_{2}, \nu_{2}\right),\left(\mu_{2}, \nu_{2}\right)\right)
$$

and $\operatorname{Inc}_{1}\left(\left(\mu_{1}, \nu_{1}\right),\left(\mu_{2}, \nu_{2}\right) \wedge\left(\mu_{3}, \nu_{3}\right)\right)=$

$$
\operatorname{Inc}_{1}\left(\left(\mu_{1}, \nu_{1}\right),\left(\mu_{2}, \nu_{2}\right)\right) \wedge \operatorname{Inc} c_{1}\left(\left(\mu_{1}, \nu_{1}\right),\left(\mu_{3}, \nu_{3}\right)\right)
$$

is not true.

Counterexample 1. $\left(\mu_{1}, \nu_{1}\right)=(0.3,0.7),\left(\mu_{2}, \nu_{2}\right)$ $=(0.2,0.5),\left(\mu_{3}, \nu_{3}\right)=(0.3,0.6)$, then

$$
\begin{gathered}
\operatorname{Inc}_{1}\left(\left(\mu_{1}, \nu_{1}\right),\left(\left(\mu_{2}, \nu_{2}\right)\right)=\frac{2}{3},\right. \\
\operatorname{Inc}_{1}\left(\left(\mu_{1}, \nu_{1}\right),\left(\mu_{3}, \nu_{3}\right)\right)=1, \\
\operatorname{Inc}_{1}\left(\left(\mu_{2}, \nu_{2}\right),\left(\mu_{3}, \nu_{3}\right)\right)=\frac{1}{2}, \\
\operatorname{Inc}_{1}\left(\left(\mu_{1}, \nu_{1}\right) \vee\left(\mu_{2}, \nu_{2}\right),\left(\mu_{3}, \nu_{3}\right)\right)=0, \\
\operatorname{Inc}_{1}\left(\left(\mu_{1}, \nu_{1}\right),\left(\mu_{2}, \nu_{2}\right) \wedge\left(\mu_{3}, \nu_{3}\right)\right)=\frac{1}{2} .
\end{gathered}
$$

It's obvious that the two equalities in Remark 1 does not hold.

Lemma 2. For any $a, b \in([0,1], \leq)$, then $\operatorname{Inc}^{\prime}(a, b)=\mathscr{I}(a, b)$ is a HM inclusion measure where $\mathscr{I}$ is a R-implicator.

Theorem 2. Let $\operatorname{Inc}^{\prime}(a, b), \operatorname{Inc}^{\prime \prime}(a, b)$ are two HM inclusion measures on $([0,1], \leq)$, then

$\operatorname{Inc}_{2}\left(\left(\mu_{1}, \nu_{1}\right),\left(\mu_{2}, \nu_{2}\right)\right)=$

$$
\mathscr{T}\left(\operatorname{Inc}{ }^{\prime}\left(\mu_{1}, \mu_{2}\right), \operatorname{Inc}^{\prime \prime}\left(1-\nu_{1}, 1-\nu_{2}\right)\right)
$$

is a HM inclusion measure on $\left(L^{*}, \leq_{L^{*}}\right)$ where $\mathscr{T}$ is a triangular norm (t-norm in short).

It can be proved easily by the properties of tnorm and R-implicator. We can get the following theorem by the same way.

Theorem 3. Let $\operatorname{Inc}^{\prime}(a, b), \operatorname{Inc}^{\prime \prime}(a, b)$ are two HM inclusion measures on $([0,1], \leq)$, then

$\operatorname{Inc}_{3}\left(\left(\mu_{1}, \nu_{1}\right),\left(\mu_{2}, \nu_{2}\right)\right)=$

$$
f\left(\operatorname{Inc}\left(\mu_{1}, \mu_{2}\right), \operatorname{Inc} c^{\prime \prime}\left(1-\nu_{1}, 1-\nu_{2}\right)\right)
$$

is a HM inclusion measure on $\left(L^{*}, \leq_{L^{*}}\right)$ where $f(x, y):[0,1] \times[0,1] \rightarrow[0,1]$ is monotone increasing function about $x$ and $y$ respectively and $f(0,0)=0, f(1,0)=0, f(1,1)=1$.

Example 2. Let $f(x, y)=\frac{x+y}{2}$, we get $\operatorname{Inc}_{3}^{\prime}\left(\left(\mu_{1}, \nu_{1}\right),\left(\mu_{2}, \nu_{2}\right)\right)=$

$$
\frac{\operatorname{Inc}^{\prime}\left(\mu_{1}, \mu_{2}\right)+\operatorname{Inc} c^{\prime \prime}\left(1-\nu_{1}, 1-\nu_{2}\right)}{2}
$$




$$
\begin{aligned}
& \operatorname{Inc}_{3}^{\prime \prime}\left(\left(\mu_{1}, \nu_{1}\right),\left(\mu_{2}, \nu_{2}\right)\right)= \\
& \quad \frac{\omega_{1} \operatorname{Inc}^{\prime}\left(\mu_{1}, \mu_{2}\right)+\omega_{2} \operatorname{Inc}^{\prime \prime}\left(1-\nu_{1}, 1-\nu_{2}\right)}{2}
\end{aligned}
$$

where $\omega_{1}, \omega_{2} \in[0,1], \omega_{1}+\omega_{2}=1$.

\subsection{Inclusion measure of IFSs}

Now we discuss the inclusion measure of IFSs based on the inclusion measure of intutionistic values. Since $(\operatorname{IFS} S(X), \leq)$ is a complete lattice, we can define a series of HM inclusion measure on $(\operatorname{IFS} s(X), \leq)$.

Theorem 4. Let $\operatorname{Inc}\left(\left(\mu_{1}, \nu_{1}\right),\left(\mu_{2}, \nu_{2}\right)\right)$ be an HM inclusion measure indicates the degree to which the intuitionistic value $\left(\mu_{1}, \nu_{1}\right)$ is contained in another intuitionistic value $\left(\mu_{2}, \nu_{2}\right)$, then

$\operatorname{Inc}_{I F 1}(A, B)=$

$$
\bigwedge_{i=1}^{n} \operatorname{Inc}\left(\left(\mu_{A}\left(x_{i}\right), \nu_{A}\left(x_{i}\right)\right),\left(\mu_{B}\left(x_{i}\right), \nu_{B}\left(x_{i}\right)\right)\right)
$$

is a HM inclusion measure of two Intuitionistic fuzzy sets $A$ and $B, A, B \in(\operatorname{IFS} S(X), \leq)$.

Proof. It's obvious that HM1 and HM2 holds;

(HM3) By the definition, $\operatorname{Inc}_{I F 1}(A, B)=$ $1 \Leftrightarrow \operatorname{Inc}\left(\left(\mu_{A}\left(x_{i}\right), \nu_{A}\left(x_{i}\right)\right),\left(\mu_{B}\left(x_{i}\right), \nu_{B}\left(x_{i}\right)\right)=1 \Leftrightarrow\right.$ $\left(\mu_{A}\left(x_{i}\right), \nu_{A}\left(x_{i}\right)\right) \leq\left(\mu_{B}\left(x_{i}\right), \nu_{B}\left(x_{i}\right)\right) \Leftrightarrow \mu_{A}\left(x_{i}\right) \leq$ $\mu_{B}\left(x_{i}\right), \nu_{A}\left(x_{i}\right) \geq \nu_{B}\left(x_{i}\right)$, for all $x_{i} \in X$, namely $A \leq$ $B$;

(HM4) $A \leq B \Leftrightarrow \mu_{A}\left(x_{i}\right) \leq \mu_{B}\left(x_{i}\right), \nu_{A}\left(x_{i}\right) \geq$ $\nu_{B}\left(x_{i}\right), \forall x_{i} \in X$; Then we have

$\operatorname{Inc}_{I F 1}(A, C)=$

$$
\begin{aligned}
& \bigwedge_{i=1}^{n} \operatorname{Inc}\left(\left(\mu_{A}\left(x_{i}\right), \nu_{A}\left(x_{i}\right)\right),\left(\mu_{C}\left(x_{i}\right), \nu_{C}\left(x_{i}\right)\right)\right) \\
\geq & \bigwedge_{i=1}^{n} \operatorname{Inc}\left(\left(\mu_{B}\left(x_{i}\right), \nu_{B}\left(x_{i}\right)\right),\left(\mu_{C}\left(x_{i}\right), \nu_{C}\left(x_{i}\right)\right)\right) \\
= & \operatorname{Inc} c_{I F 1}(B, C) ;
\end{aligned}
$$

By the same way, we get $\operatorname{Inc}_{I F 1}(C, A) \leq$ $\operatorname{Inc}_{I F 1}(C, B)$.

So $\operatorname{Inc}_{I F 1}(A, B)$ is a $\mathrm{HM}$ inclusion measure.

By the same way, we can get the following theorems:

Theorem 5. Let $\operatorname{Inc}\left(\left(\mu_{1}, \nu_{1}\right),\left(\mu_{2}, \nu_{2}\right)\right)$ be an HM inclusion measure indicates the degree to which the intuitionistic value $\left(\mu_{1}, \nu_{1}\right)$ is contained in another intuitionistic value $\left(\mu_{2}, \nu_{2}\right)$, then

$\operatorname{Inc}_{I F 2}(A, B)=$

$$
\sum_{i=1}^{n} \lambda_{i} \operatorname{Inc}\left(\left(\mu_{A}\left(x_{i}\right), \nu_{A}\left(x_{i}\right)\right),\left(\mu_{B}\left(x_{i}\right), \nu_{B}\left(x_{i}\right)\right)\right)
$$

is a HM inclusion measure of two Intuitionistic fuzzy sets $A$ and $B, A, B \in(\operatorname{IFSs}(X), \leq)$, where $\sum_{i=1}^{n} \lambda_{i}=1, \lambda_{i} \geq 0$.

Theorem 6. Let $\operatorname{Inc}\left(\left(\mu_{1}, \nu_{1}\right),\left(\mu_{2}, \nu_{2}\right)\right)$ be an HM inclusion measure indicates the degree to which the intuitionistic value $\left(\mu_{1}, \nu_{1}\right)$ is contained in another intuitionistic value $\left(\mu_{2}, \nu_{2}\right)$, then

$\operatorname{Inc}_{I F 3}(A, B)=$

$$
\begin{aligned}
& f\left(\operatorname{Inc}\left(\left(\mu_{A}\left(x_{1}\right), \nu_{A}\left(x_{1}\right)\right),\left(\mu_{B}\left(x_{1}\right), \nu_{B}\left(x_{1}\right)\right)\right), \ldots,\right. \\
& \left.\operatorname{Inc}\left(\left(\mu_{A}\left(x_{n}\right), \nu_{A}\left(x_{n}\right)\right),\left(\mu_{B}\left(x_{n}\right), \nu_{B}\left(x_{n}\right)\right)\right)\right)
\end{aligned}
$$

is a HM inclusion measure of two Intuitionistic fuzzy sets $A$ and $B, A, B \in(\operatorname{IFSs}(X), \leq)$ where $f:[0,1]^{n} \rightarrow[0,1]$ is increasing multi-variable function about every variable and $\mathrm{f}(0, \ldots, 0)=1$.

Theorem 7. For any $A, B \in(\operatorname{IFSs}(X), \leq$ )$, \operatorname{Inc}_{I F}(A, B), \operatorname{Inc}_{I F}^{\prime}(A, B)$ are two HM inclusion measures of intuitionistic fuzzy sets, then the following mappings are also HM inclusion measure of intuitionistic fuzzy sets:

(1) $\operatorname{Inc}_{I F 4}(A, B)=\operatorname{Inc}_{I F}(A, B) \wedge \operatorname{Inc} c_{I F}\left(\left(B^{c}, A^{c}\right)\right.$;

(2) $\operatorname{Inc}_{I F 5}(A, B)=\operatorname{Inc} c_{I F}(A, B) \cdot \operatorname{Inc} c_{I F}\left(B^{c}, A^{c}\right)$;

(3) $\operatorname{Inc}_{I F 6}(A, B)=\operatorname{Inc} c_{I F}(A, B) \wedge \operatorname{Inc} c_{I F}^{\prime}(A, B)$;

(4) $\operatorname{Inc}_{I F 7}(A, B)=\mathscr{T}\left(\operatorname{Inc}_{I F}(A, B), \operatorname{Inc}_{I F}^{\prime}(A, B)\right)$;

( $\mathscr{T}$ is a t-norm)

(5) $\operatorname{Inc}_{I F 8}(A, B)=\frac{\operatorname{Inc}_{I F}(A, B)+\operatorname{Inc} c_{I F}^{\prime}(A, B)}{2}$.

\section{Similarity measure between IFSs}

In this section, we discuss the similarity measure between IFSs based on the inclusion measure. Definition 6. Let $(L, \leq)$ be a complete lattice, we denote the mapping $S: L \times L \rightarrow[0,1]$ as a $\mathbb{S}$ similarity measure on $\mathrm{L}$, such that the value $S(a, b)$ will indicate the measure of similatity between two elements of $\mathrm{L}$ and satisfies the following conditions:

(S1) $0 \leq S(a, b) \leq 1, \forall a, b \in L$;

(S2) $S(a, b)=1 \Leftrightarrow a=b, \forall a, b \in L$;

(S3) $S(a, b)=S(b, a), \forall a, b \in L$;

(S4) If $a, b, c \in L$ and $a \leq b \leq c$, then $S(a, c) \leq$ $\operatorname{Inc}(a, b)$ and $S(a, c) \leq S(b, c)$.

(S5) $S(a, b)=0$ if $a=[1], b=[0]$ or $a=[0], b=$ [1], where [1] and [0] are the maximal element and minimum element respectively.

Theorem 8. For all $a, b \in L$, Let Inc $(\mathrm{a}, \mathrm{b})$ is a HM inclusion measure on the complete lattice $\mathrm{L}$, then $S(a, b)=\mathscr{T}(\operatorname{Inc}(a, b), \operatorname{Inc}(b, a))$ is a $\mathbb{S}$ similarity measure where $\mathscr{T}$ is a t-norm.

Proof. It's obvious that S1 and S3 hold;

(S2) By the monotonic increasing property of t-norm and property of HM inclusion measure, 
we have $S(a, b)=\mathscr{T}(\operatorname{Inc}(a, b), \operatorname{Inc}(b, a))=1 \Leftrightarrow$ $\operatorname{Inc}(a, b)=\operatorname{Inc}(a, b)=1 \Leftrightarrow a \leq b, b \leq a \Leftrightarrow a=b ;$

(S4) If $a, b, c \in L$ and $a \leq b \leq c$, then by the definition of t-norm and HM inclusion measure,

$$
\begin{aligned}
S(a, b)=\mathscr{T}(\operatorname{Inc}(a, b), \operatorname{Inc}(b, a)) & =\mathscr{T}(1, \operatorname{Inc}(b, a)) \\
& =\operatorname{Inc}(b, a) ;
\end{aligned}
$$

By the similar way, $S(a, c)=\operatorname{Inc}(c, a), S(b, c)=$ $\operatorname{Inc}(c, b)$; So we get $S(a, c) \leq S(a, b)$ and $S(a, c) \leq$ $S(b, c)$ by the monotonicity of HM inclusion measure.

(S5)By the monotonic increasing property of t-norm and definition of $\mathrm{HM}$ inclusion measure, we get $S([1],[0])=S([0],[1])=$ $\mathscr{T}(\operatorname{Inc}([0],[1]), \operatorname{Inc}([1],[0]))=0$.

Therefore $S(a, b)=\mathscr{T}(\operatorname{Inc}(a, b), \operatorname{Inc}(b, a))$ is a $\mathbb{S}$ similarity measure.

As for the similarity measure between the intuitionistic values and IFSs, we have the following parallel theorems:

Theorem 9. Let $\left(\mu_{1}, \nu_{1}\right),\left(\mu_{2}, \nu_{2}\right) \in\left(L^{*}, \leq_{L^{*}}\right)$ be two intuitionistic values, $\operatorname{Inc}\left(\left(\mu_{1}, \nu_{1}\right),\left(\mu_{2}, \nu_{2}\right)\right)$ is a $\mathrm{HM}$ inclusion measure on the complete lattice $\left(L^{*}, \leq_{L^{*}}\right)$, then $S\left(\left(\mu_{1}, \nu_{1}\right),\left(\mu_{2}, \nu_{2}\right)\right)=$

$$
\mathscr{T}\left(\operatorname{Inc}\left(\left(\mu_{1}, \nu_{1}\right),\left(\mu_{2}, \nu_{2}\right)\right), \operatorname{Inc}\left(\left(\mu_{2}, \nu_{2}\right),\left(\mu_{1}, \nu_{1}\right)\right)\right)
$$

is a $\mathbb{S}$ similarity measure between two intuitionistic values where $\mathscr{T}$ is a t-norm.

Theorem 10. For all $A, B \in(\operatorname{IFS} S(X), \leq)$, Let $\operatorname{Inc}(\mathrm{A}, \mathrm{B})$ is a HM inclusion measure on the complete lattice $(\operatorname{IFS} S(X), \leq)$, then $S(A, B)=$ $\mathscr{T}(\operatorname{Inc}(A, B), \operatorname{Inc}(B, A))$ is a $\mathbb{S}$ similarity measure between two intuitionistic fuzzy sets where $\mathscr{T}$ is a t-norm.

Remark 3. Y.H Li et al. gave examples to show that the condition S5 is necessary to the definition of similarity measure between IFSs. Now a series of reasonable similarity measures are given which satisfy the five conditions S1-S5.

\section{Conclusion}

In this paper, we have discussed the definitions of HM inclusion measure and $\mathbb{S}$ similarity measure according to the axiomatic and practical demand; Some formulas have been given to calculate the HM inclusion measure and similarity measure; We think they will be useful to approximate reasoning, pattern recognition and so on.

\section{Acknowledgement}

This work is partially supported by the Natural Science Foundation of the Education Department of Shaanxi Province of China (Grant No. 07JK422) and the Social Science Foundation of the Education Department of Shaanxi Province of China (Grant No. 07JK101).

\section{References}

[1] L.A. Zadeh, Fuzzy sets. Information and Control, 8:338-353, 1965.

[2] K.T. Atanassov, Intuitionistic fuzzy sets. VI ITKR's Session, Sofia deposed in Central Sci-Technical Library of Bulg. Acad. of Sci., 84:1697, 1983.

[3] L.A. Zadeh, Outline of a new approach to the analysis of complex systems and decision processes with interval-valued fuzzy sets. IEEE Transaction on Systems, Man and Cybernetics, 109:453-457, 2000.

[4] J.E Hayes, D.Michie, L.I. Mikulich, Theory of approximate reasoning. Ed, Machine Intelligence, pp.149-194, 1970.

[5] I.B. Turksen, Interval valued fuzzy sets based on normal forms. Fuzzy Sets and Systems, 20:191210, 1986.

[6] S.K. De, R. Biswas, R. A., An application of intuitionistic fuzzy set in medical diagnosis. Fuzzy Sets and Systems, 117:209-213, 2001.

[7] M.B. Gorzalczany, A method of inference in approximate reasoning based on interval-valued fuzzy sets. Fuzzy Sets and Systems, 21:1-17, 1987.

[8] H. Bustince, Indicator of inclusion grade for interval-valued fuzzy sets. Application to approximate reasoning based on interval-valued fuzzy sets. International Journal of Approximate Reasoning, 23:137-209, 2000.

[9] I.B. Turksen, Z. Zhong, An approximate analogical reasoning schema based on similarity measures and interval-valued fuzzy sets. Fuzzy Sets and Systems, 34:323-346, 1990.

[10] C. Cornelis, G. Deschrijver, E.E. Kerre, Implication in intuitionistic fuzzy and interval-valued fuzzy set theory: construction, classification, application. International Journal of Approximate Reasoning, 35:55-95, 2004.

[11] W. Bandler, L. Kohout, Fuzzy power sets and fuzzy implication operators. Fuzzy Sets and Systems, 4:13-30, 1980. 
[12] R. Willmott, Two fuzzier implication operators in the theory of fuzzy power sets. Fuzzy Sets and Systems, 4:31-36, 1980.

[13] W.X. Zhang, Y. Leung, The Uncertainty Reasoning Principles, Xi'an Jiaotong University Press, Xi'an, 1996.

[14] W.X. Zhang, Y. Leung, Theory of including degrees and its applications to uncertainty inferences. Soft Computing in Intelligent Systems and Information Processing, New York: IEEE, pp.496-501, 1996.

[15] D. Sinha, E.R. Dougherty, Fuzzication of set inclusion: theory and applications. Fuzzy Sets and Systems, 55:15-42, 1993.

[16] V.R. Young, Fuzzy subsethood. Fuzzy Sets and Systems, 77: 371-384, 1996.

[17] C. Cornelis, C.V. Donck, E.E. Kerre, Sinhadougherty approach to the fuzzication of set incluaion revisited. Fuzzy Sets and Systems, 134:283-295, 2003.

[18] C.P. Pappis, Value approximation of fuzzy systems variables. Fuzzy Sets and Systems, 39:111115, 1991.

[19] C.P. Pappis, N.I. Karacapilidis, Application of a similarity measure of fuzzy sets to fuzzy relational equations. Fuzzy Sets and Systems, 75:135-142, 1992.

[20] C.P. Pappis, N.I. Karacapilidis, A comparative assessment of measures of similarity of fuzzy values. Fuzzy Sets and Systems, 56:171174, 1993.

[21] X.C. Liu, Entropy, distance measure and similarity measure of fuzzy sets and their relations. Fuzzy Sets and Systems, 52:305-318, 1992.

[22] J. Fan, W. Xie, Some notes on similarity measure and proximity measure, Fuzzy Sets and Systems, 101:403-412, 1999.

[23] W.J. Wang, B.D. Baets, E.E. Kerre, A comparative study of similarity measures. Fuzzy Sets and Systems, 73:259-268, 1995.

[24] H. Bustince, Indicator of inclusion grade for interval-valued fuzzy sets. Application to approximate reasoning based on interval-valued fuzzy sets, International Journal of Approximate Reasoning, 23:137-209, 2000.

[25] C. Cornelis, E.E. Kerre, Inclusion measures in the Intuitionistic fuzzy sets. ECSQARU 2003, Lecture Notes in Atificial Intelligence 2711, pp.345-356, 2003.

[26] P. Grzgorzewski and E. Mrowka, Subsethood measure of intuitionistic fuzzy sets. FUZZIEEE 2004, pp.139-142, 2004.

[27] J. H. Park, J. S. Park, Y. C. Kwun, On fuzzy inclusion in the interval-valued sense. FSKD
2005, Lecture Notes in Atificial Intelligence 3613, pp.1-10, 2005.

[28] C.Y. Zhang, H.Y. Fu, Similarity measures on three kinds of fuzzy sets. Pattern Recognition Letters, 27:1307-1317, 2006.

[29] Y.H. Li, David L.Olson, Q. Zheng, Similarity measures between intuitionistic fuzzy (vague) sets: A comparative analysis. Pattern Recognition Letters, 28:278-285, 2007.

[30] P. Burillo, H. Bustince, Entropy on intuitionistic fuzzy sets and on interval-valued fuzzy sets. Fuzzy Sets and Systems, 78:305-316, 1996.

[31] K.T. Atanassov, G. Gargov, Interval valued intuitionistic fuzzy sets. Fuzzy Sets and Systems, 31:343-349, 1989.

[32] J.L. Fan, W. Xie, J. Pei, Subsethood measure: new definitions. Fuzzy Sets and Systems, 106:201-209, 1999. 